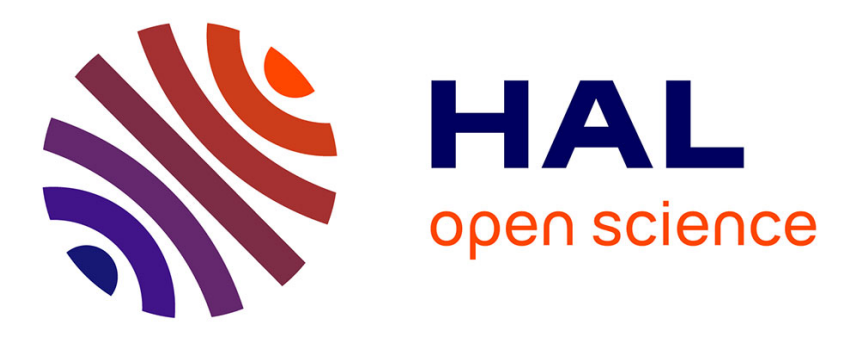

\title{
Characterization of muscle belly elastic properties during passive stretching using transient elastography
}

Antoine Nordez, Jean Luc Gennisson, Pascal Casari, Stefan Catheline, Christophe Cornu

\section{- To cite this version:}

Antoine Nordez, Jean Luc Gennisson, Pascal Casari, Stefan Catheline, Christophe Cornu. Characterization of muscle belly elastic properties during passive stretching using transient elastography. Journal of Biomechanics, 2008, 41 (10), pp.2305-2311. 10.1016/j.jbiomech.2008.03.033 . hal-01005849

\section{HAL Id: hal-01005849 \\ https://hal.science/hal-01005849}

Submitted on 22 Nov 2018

HAL is a multi-disciplinary open access archive for the deposit and dissemination of scientific research documents, whether they are published or not. The documents may come from teaching and research institutions in France or abroad, or from public or private research centers.
L'archive ouverte pluridisciplinaire HAL, est destinée au dépôt et à la diffusion de documents scientifiques de niveau recherche, publiés ou non, émanant des établissements d'enseignement et de recherche français ou étrangers, des laboratoires publics ou privés. 


\title{
Characterization of muscle belly elastic properties during passive stretching using transient elastography
}

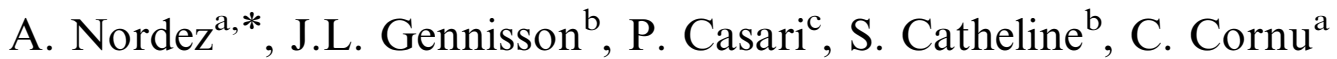 \\ ${ }^{a}$ Université de Nantes, Nantes Atlantique Universités, Laboratoire "Motricité, Interactions, Performance”, JE 2438, UFR STAPS, \\ 25 bis Bd Guy Mollet, BP 72206, Nantes F-44000, France \\ ${ }^{\mathrm{b}}$ Laboratoire Ondes et Acoustique, ESPCI, UMR CNRS 7587, INSERM, Université Paris VII, 10 rue Vauquelin, 75231 Paris Cedex 05, France \\ ${ }^{\mathrm{c}}$ Université de Nantes, Nantes Atlantique Universités, Institut de Recherche en Génie Civil et Mécanique, CNRS, UMR 6183, UFR des Sciences et Techniques, \\ 2 rue de la Houssinière, BP 92208, Nantes F-44000 France
}

\begin{abstract}
Passive muscle stretching can be used in vivo to assess the viscoelastic properties of the entire musculo-articular complex, but does not allow the specific determination of the muscle or tendon viscoelasticity. In this respect, the local muscle hardness (LMH) of the gastrocnemius medialis (GM) belly was measured during a passive ankle stretching of 10 subjects using transient elastography. A Biodex isokinetic dynamometer was used to stretch ankle plantar flexors, to measure ankle angle, and the passive torque developed by the ankle joint in resistance to the stretch. Results show that the LMH increased during the stretching protocol, with an averaged ratio between maximal LMH and minimal LMH of $2.62 \pm 0.46$. Furthermore, $\mathrm{LMH}$-passive torque relationships were nicely fitted using a linear model with mean correlation coefficients $\left(R^{2}\right)$ of $0.828 \pm 0.099$. A good reproducibility was found for the maximal passive torque (ICC $=0.976$, $\mathrm{SEM}=2.9 \mathrm{Nm}, \mathrm{CV}=5.5 \%)$ and the $y$-intercept of the $\mathrm{LMH}-$ passive torque relationship $(\mathrm{ICC}=0.893, \mathrm{SEM}=105 \mathrm{~Pa}, \mathrm{CV}=7.8 \%)$. However, the reproducibility was low for the slope of this relationship (ICC $=0.631, \mathrm{SEM}=10.35 \mathrm{~m}^{-2}, \mathrm{C} \mathrm{V}=60.4 \%$ ). The $y$-intercept of the LMH-passive torque relationship was not significantly changed after 10 min of static stretching. This result confirms the finding of a previous study indicating that changes in passive torque following static stretching could be explained by an acute increase in muscle length without any changes in musculo-articular intrinsic mechanical properties.
\end{abstract}

Keywords: Muscle stiffness; Static stretching; Passive torque; Transient elastography

\section{Introduction}

Many studies have shown that the characterization of muscle mechanical properties enables clinicians and researchers to have a better understanding of muscle function and of the mechanisms responsible for muscle adaptations following acute or chronic solicitations (e.g. Goubel and Lensel-Corbeil, 2003). Experimental techniques classically used to study the elastic behavior of muscle in vivo, such as quick release (e.g. Cornu et al., 1998; Hof, 1998; Pousson et al., 1990) or sinusoidal perturbations (e.g. Cornu et al., 2001; Lambertz et al., 2001; Winters et al., 1988), enable the assessment of global mechanical proper-

\footnotetext{
${ }^{*}$ Corresponding author. Tel.: + 33251837217 ; fax: + 33251837210 .

E-mail address: antoine.nordez@univ-nantes.fr (A. Nordez).
}

ties of the muscle tendon unit or the musculo-articular complex without any differentiation of the involved structures. Therefore, the use of more localized techniques could be quite valuable to measure separately the mechanical properties of muscle and tendon. For this purpose, in the last few years, ultrasonography has enabled researchers to assess the mechanical properties of specific local structures such as tendon (e.g. Hansen et al., 2006; Kubo et al., 2001; Maganaris and Paul, 1999; Reeves et al., 2003). However, ultrasonography investigations are limited to superficial structures and do not easily allow for an assessment of specific muscle mechanical properties.

In fact, the noninvasive assessment of localized muscle mechanical properties would enable the estimation of individual muscle contributions to global joint stiffness, and remains a topic of continued interest for researchers. 


\section{Nomenclature}

CV coefficient of variation

ROM range of motion

GL gastrocnemius lateralis

SEM standard error of the measurement

GM gastrocnemius medialis

sEMG surface electromyography

ICC intra-class correlation coefficient
SO

LMH local muscle hardness

SSI supersonic shear imaging

MRE magnetic resonance Elastography

$V_{\mathrm{S}} \quad$ shear wave velocity

$R^{2} \quad$ determination coefficient

$\rho \quad$ muscle mass density

RMS root mean square

$\mu \quad$ elastic shear modulus
While all of the above studies concern the assessment of the stiffness along the longitudinal axis of muscle and tendon, another approach called transient elastography has been developed to characterize a longitudinal or transverse muscle stiffness that has been termed "hardness" (Gennisson et al., 2003). More precisely, using ultrasound imaging, transient elastography consists of following the shear wave propagation and measuring their velocity, which is related to the LMH (Gennisson et al., 2003; Sandrin et al., 2002a, b) (Eq. (1)):

$\mathrm{LMH}=\mu=\rho V_{\mathrm{S}}^{2}$

where $\rho$ is the muscle mass density $\left(\rho \approx 1000 \mathrm{~kg} \mathrm{~m}^{-3}\right), \mu$ the elastic shear modulus and $V_{\mathrm{S}}$ the shear wave velocity.

This technique would allow the assessment of hardness of both superficial and deep structures. Thus, a few studies using elastographic methods have shown that the in vivo human LMH is increased with the level of contraction (Gennisson et al., 2005; Heers et al., 2003; Jenkyn et al., 2003; Levinson et al., 1995). Moreover, other methods of elastography could also be used to determine LMH in passive conditions. For example, using magnetic resonance elastography (MRE), Dresner et al. (2001) have shown that the LMH is linearly increased with the passive tension for in vitro beef muscles. These results are in agreement with those of Jenkyn et al. (2003) who have shown using MRE in vivo that the LMH of plantar flexors is ankle-angle dependant. However, since MRE implies performing static experiments with an acquisition time of about $1 \mathrm{~min}$ (Jenkyn et al., 2003), the passive LMH cannot be measured in real time during passive muscle lengthening in vivo. Conversely, transient elastography allows the LMH measurement in $50 \mathrm{~ms}$ and could then be proposed as a suitable method to follow LMH changes during a stretching protocol.

It has been shown in the literature that passive stretching protocols induce an acute decrease in the passive torque developed by the musculo-articular complex in resistance to stretch (Magnusson et al., 1998, 1996; McNair et al., 2001, 2002; Nordez et al., 2006, in press). Previous research has shown that dissipative properties (i.e. the normalized area of torque-angle relationship hysteresis) are not changed after static stretching (Nordez et al., in press). Additionally, it has been shown by using in vitro rabbit muscle that static stretching induces an acute increase in muscle length (Taylor et al., 1990). Therefore, it could be hypothesized that changes in passive torque are explained by acute increases in muscle length, without any changes in their intrinsic mechanical properties. This kind of hypothesis could be verified using transient elastography and it would provide fundamental data to better understand the mechanical basis of stretching.

Using transient elastography, in order to assess acute or chronic effects on the $\mathrm{LMH}$, implies that reliable measurements are possible. In fact, even if previous studies have shown variability in the measurements performed in vivo using elastography (Gennisson et al., 2005; Heers et al., 2003; Jenkyn et al., 2003; Levinson et al., 1995), to our knowledge, the reliability of the LMH measure provided by transient elastography has never been determined.

Therefore, the aims of the present study were (i) to assess the relationships between LMH measured using transient elastography, angle and passive torque during one passive stretching test performed in vivo; (ii) to determine the reliability of such relationships; and (iii) to assess changes in passive LMH induced by a prolonged passive static stretching protocol.

\section{Materials and methods}

\subsection{Subjects}

Nine healthy male subjects $(25 \pm 3$ years, height: $183.5 \pm 7.5 \mathrm{~cm}$, weight: $75.3 \pm 8.3 \mathrm{~kg}$ ) volunteered to participate for the present study and signed an informed consent form. This study was conducted according to the Helsinki statement (1964).

\subsection{Measurement techniques}

The Biodex system 3 research $^{\circledR}$ (Biodex medical, Shirley, NY, USA) isokinetic dynamometer was used to measure ankle angle and the passive torque produced by the ankle joint in resistance to the stretch. The position of the subjects on the dynamometer was similar to the position described in previous studies (Kubo et al., 2001; Riemann et al., 2001). Briefly, subjects were lying prone on the dynamometer and their right leg was fully extended. The input axis of the dynamometer was aligned with the supposed axis of rotation of the right ankle (Fig. 1).

Bipolar surface electromyographic (sEMG) signals were recorded from surface electrodes $(\mathrm{Ag} / \mathrm{AgCl}, 4 \mathrm{~mm}$ recording diameter, In Vivo Metric, 
A

B

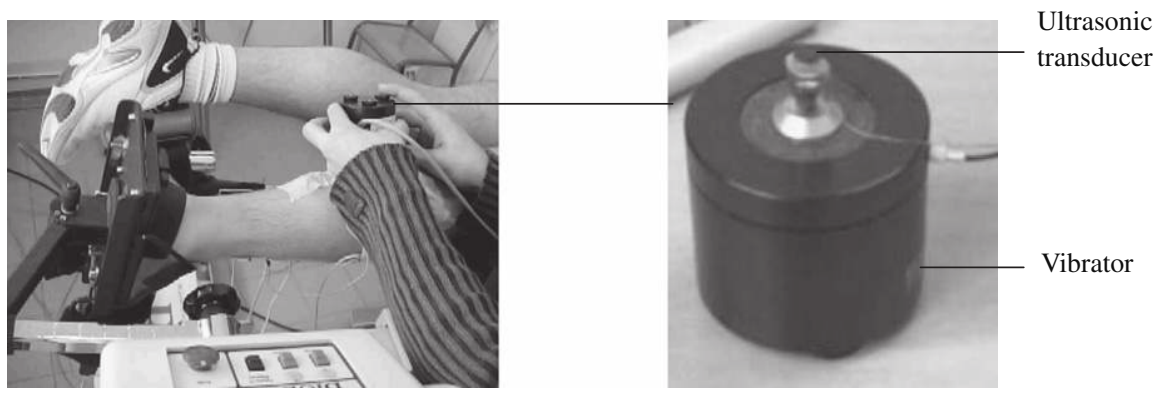

Fig. 1. Measurement techniques. (A) Position of the subject on the dynamometer: subjects were lying prone, and their right knee was fully extended. The reference ankle angle $\left(0^{\circ}\right)$ was the foot perpendicular to the leg with positive angles in dorsiflexion. (B) The shear elasticity probe composed of a vibrator and an ultrasonic transducer.

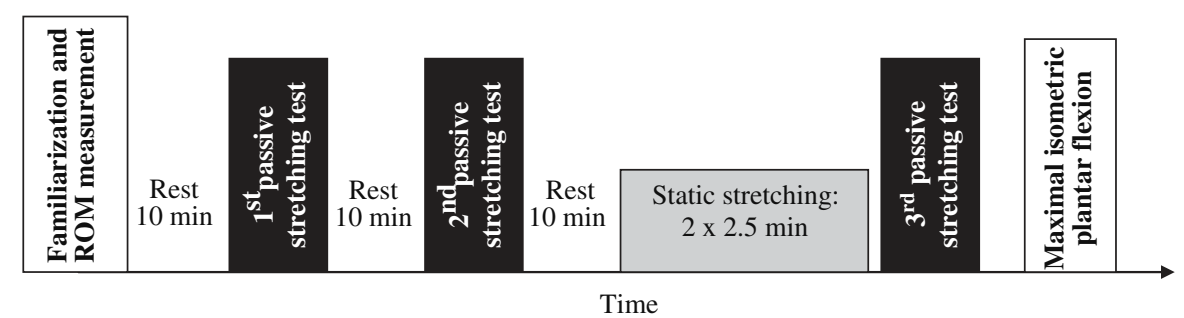

Fig. 2. Experimental protocol. The familiarization was followed by two passive stretching tests (pre-test) until $95 \%$ of the maximal range of motion $(\mathrm{ROM})$ at $0.5^{\circ} \mathrm{s}^{-1}$. Thereafter, a third identical passive stretching test (post-test) was performed after a static stretching protocol. During the three passive stretching tests, local muscle hardness (LMH) measurements were performed each $2.5 \mathrm{~s}$. Finally, subjects performed three maximal isometric plantar flexions in order to normalize sEMG data.

Healdsburg, CA, USA) placed on the gastrocnemius lateralis (GL), GM and soleus (SO) muscles with an $11 \mathrm{~mm}$ interelectrode distance. According to the surface electromyography for the noninvasive assessment of muscles (SENIAM) recommendations (Hermens et al., 2000), surface electrodes were placed with respect to the assumed direction of the fibers. Three reference electrodes were placed over the lateral and medial malleolus. The interelectrode resistance was reduced to less than $55 \mathrm{k} \Omega$ (Hewson et al., 2003) after standard skin preparation procedures: shaving, slight abrasion and cleaning with an alcohol-acetone solution. sEMG signals were preamplified $($ gain $=600$ ).

In order to assess LMH, the shear elasticity probe (Fig. 1B) (Gennisson et al., 2005; Sandrin et al., 2002b) was placed at the middle of GM muscle belly close to surface electrodes. Briefly, it was composed of an ultrasonic transducer (central frequency: $5 \mathrm{MHz}$; diameter: $7 \mathrm{~mm}$; focal depth: $35 \mathrm{~mm}$; Vermon, Tours, France) mounted on a mini shaker (Brüel \& Kjær, type 4810, DK-2850 Nærum, Denmark). The vibrator impacted GM muscle (low-frequency sinusoidal pulse: $100 \mathrm{~Hz}$, $1 \mathrm{~mm}$ amplitude) with the front face of the transducer working in emission-reception. The transducer worked as a pulse-echo system with a $2 \mathrm{kHz}$ recurrence frequency. Ultrasonic signals $(5 \mathrm{MHz}$ central frequency) were sampled at $50 \mathrm{MHz}$ and stored on a computer hard drive using a 9-bit digitizer with $2 \mathrm{MB}$ memory. The low-frequency pulse propagates within the medium as shear waves. With ultrasound, shear wave displacements were retrieved from a cross-correlation algorithm (Ophir et al., 1991). From the displacement value, the shear velocity is calculated and directly provides the LMH (Gennisson et al., 2005; Sandrin et al., 2002b). LMH measurements were performed in less than $50 \mathrm{~ms}$.

The passive torque, the ankle angle, the low-frequency sinusoidal pulse sent with the conventional function generator (for synchronization purposes between LMH and sEMG measurements) and sEMG signals were sampled at 256, 512 and $1024 \mathrm{~Hz}$, respectively, with a 12-bit analogic/ digital converter (Myodata ${ }^{\circledR}$, Electronique du Mazet, France, input impedance $=10 \mathrm{G} \Omega$, common mode-rejection ratio at $50 \mathrm{~Hz}=100 \mathrm{~dB}$ ).
Data were finally stored in a flash memory card and transferred to a computer hard drive for further analysis.

\subsection{Experimental protocol}

The experimental protocol is described in Fig. 2. A familiarization protocol with the passive stretching test was first performed and the maximal range of motion (ROM) in dorsi flexion was first measured (Nordez et al., 2006). sEMG signals were visualized in real time by the experimenter and the subject. After a $10 \mathrm{~min}$ rest period, a passive stretching test (pre-test) was imposed at a very slow velocity $\left(0.5^{\circ} \mathrm{s}^{-1}\right)$ from $40^{\circ}$ in plantar flexion until $95 \%$ of the maximal ROM. A pilot study has shown that after a familiarization session most of our subjects were able to relax until this ROM. Furthermore, the data were discarded when activity levels exceeded $1 \%$ of the level reached during the maximal isometric contractions (McNair et al., 2001, 2002). LMH measurements were performed every $2.5 \mathrm{~s}$ during the passive stretching test. Then, after a $10 \mathrm{~min}$ rest period, subjects repeated the same passive stretchings test to assess the reliability of the measurements. Thereafter, after an additional rest period of $10 \mathrm{~min}$, subjects performed four 150 s passive static stretching at $80 \%, 82 \%, 84 \%$ and $86 \%$ of the maximal ROM. The static stretching was immediately followed by a third identical passive stretching test (post-test) in order to assess the effects of the static stretching protocol. Finally, the three maximal isometric plantar flexions were performed with a $45 \mathrm{~s}$ rest period in between

\subsection{Data processing and statistics}

Using inversion algorithm developed by Catheline et al. (2004) in soft solids and by Gennisson et al. (2005) in muscles, it has been possible to estimate the LMH. The general idea consists in computing the shear wave speed across GM muscle depth using the Helmholtz equation (see Eq. (2)). The time and spatial numerical derivatives were calculated from the displacement field obtained with transient elastography. Then, the LMH 

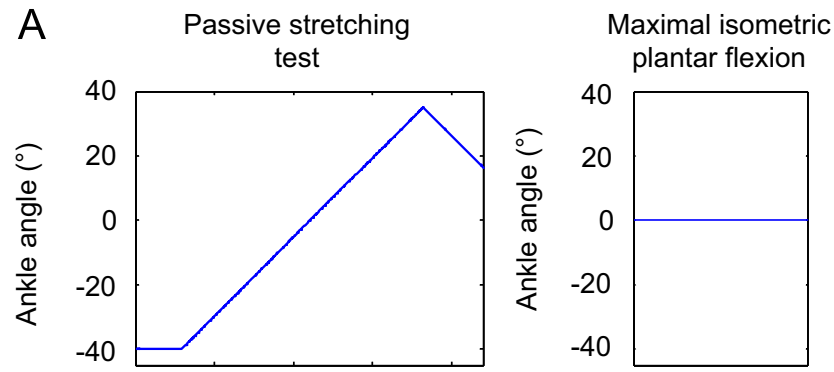

B
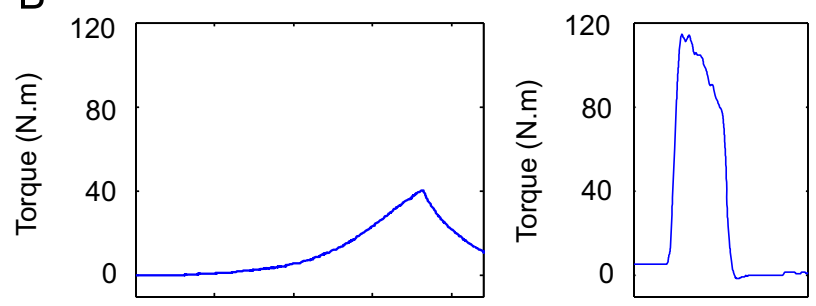

C
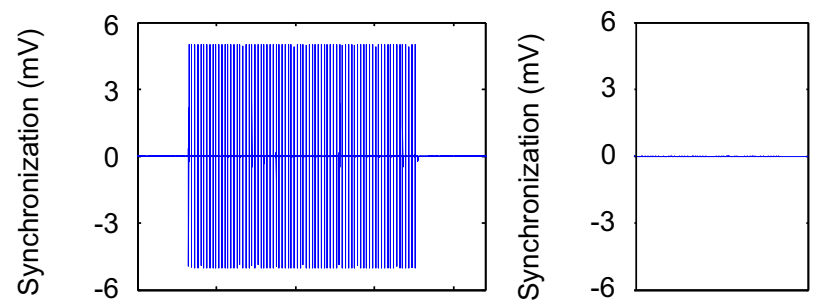

D
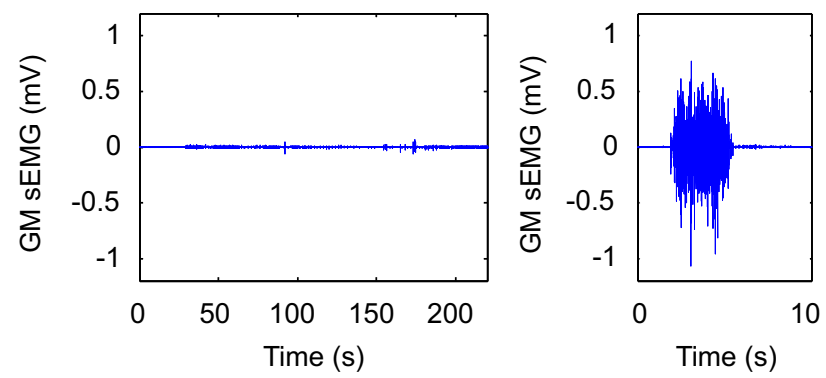

Fig. 3. Typical raw data collected during a passive stretching test (left graphs) and a maximal isometric plantar flexion (right graphs). (A)- ankle angle; (B)- torque measured at the ankle joint; (C)- sinusoidal pulses sent using the function generator (one pulse corresponded to one LMH measure) used to synchronize LMH and torque measurements (D)surface electromyographic (sEMG) signal of gastrocnemius medialis (GM).

was deduced from Eq. (1):

$\frac{\partial^{2} \vec{u}}{\partial t^{2}}-\frac{1}{V_{\mathrm{S}}^{2}} \frac{\partial^{2} \vec{u}}{\partial x^{2}}=0$

where $\vec{u}$ is displacement; $t$ is time and $x$ is muscle depth.

Torque and sEMG signals were filtered using dual low-pass $(10 \mathrm{~Hz})$ and band-pass $(6-400 \mathrm{~Hz})$ second-order Butterworth filters, respectively. Typical raw data are shown in Fig. 3. The time corresponding to LMH measurements was determined using the low-frequency sinusoidal pulse sent with the conventional function generator. For each LMH measurement, the passive torque, ankle angle and the root mean square (RMS) of sEMG signals (using a $200 \mathrm{~ms}$ window placed around the pulse) were determined. Thus, torque-angle, $\mathrm{LMH}$-angle and $\mathrm{LMH}$-torque relationships were assessed. Since we were only interested in plantar flexors stretch, all the measurements obtained for negative torque values (dorsiflexion passive torque) were not taken into consideration. In addition, LMH measurements in which RMS of sEMG signal exceeded $1 \%$ of the maximal level reached during the maximal isometric plantar flexion were discarded (McNair et al., 2001, 2002).

A pilot study has shown that a linear fit could be used to model LMH changes with passive torque. The determination coefficient $\left(R^{2}\right)$ and the $P$ value of the fit were determined for each relationship. The reliability of the maximal passive torque, $y$-intercept and slope of LMH-torque relationships was assessed using an intraclass correlation coefficient (ICC), standard error of the measurement (SEM) and coefficient of variation (CV) (Hopkins, 2000). A paired $t$-test was used to compare pre- and posttest values for reproducible parameters. The critical level of significance was set at $P<0.05$.

\section{Results}

One subject was excluded from the study because sEMG RMS levels were higher than the criterion during most of the passive stretching tests.

\subsection{LMH-angle and LMH-torque relationships}

Typical LMH-torque relationships obtained during passive stretching test are described in Fig. 4. Individual $R^{2}$ values are also reported in the Table 1 , and indicated that the linear model was nicely fitted to this relationship $\left(R^{2}=0.812 \pm 0.080,0.69-0.93, P<0.001\right)$. Therefore, the LMH was increased with the stretching level, and the mean ratio between maximal and minimal $\mathrm{LMH}\left(\mathrm{LMH}_{\max } /\right.$ $\left.\mathrm{LMH}_{\min }\right)$ was about $2.5\left(\mathrm{LMH}_{\max } / \mathrm{LMH}_{\min }=2.62 \pm 0.46\right.$, 1.84-3.08).

\subsection{Reliability}

Two typical examples of LMH-torque relationships are shown in Fig. 4. For three subjects, results obtained for the two measurements were similar (Fig. 4A), while for five subjects, LMH measurements were clearly different for high levels of passive torque (Fig. 4B). Overall, the reliability was good for the maximal passive torque $(\mathrm{ICC}=0.976, \mathrm{SEM}=2.9 \mathrm{Nm}, \mathrm{CV}=5.4 \%)$ and the $y$ intercept of the LMH-torque relationship $(\mathrm{ICC}=0.893$, $\mathrm{SEM}=105 \mathrm{~Pa}, \mathrm{CV}=7.8 \%$ ), while it was low for the slope of this relationship $\left(\mathrm{ICC}=0.631, \mathrm{SEM}=10.35 \mathrm{~m}^{-2}\right.$, $\mathrm{CV}=60.4 \%)$.

\subsection{Effects of static stretching}

The passive torque was significantly $(P<0.01)$ decreased after the static stretching protocol from $57.6 \pm 18.0$ to $50.6 \pm 16.6 \mathrm{Nm}(-12.2 \pm 8.9 \%)$. Conversely, no significant change in the $y$-intercept of the LMH-torque relationship was found (pre-test: $1000 \pm 351 \mathrm{~Pa}$; post-test: $1012 \pm 382 \mathrm{~Pa}$; $P>0.05)$. 
A

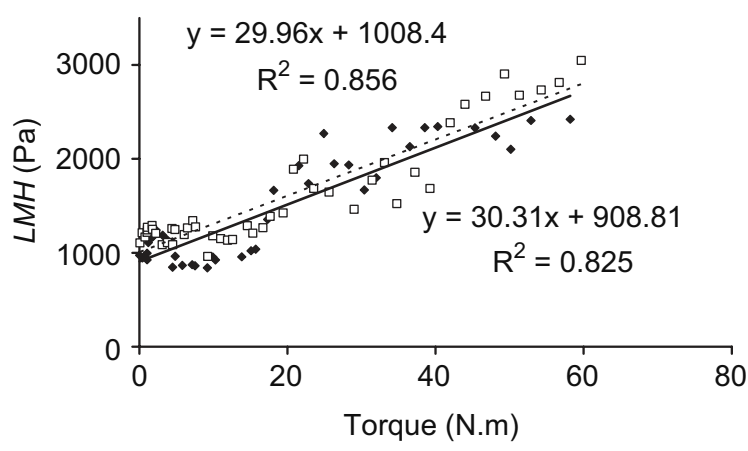

B

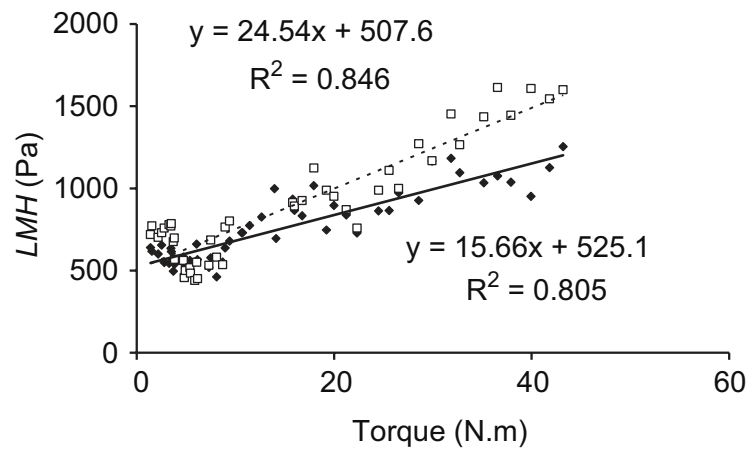

Fig. 4. Typical examples of relationships between local muscle hardness (LMH) measured using transient elastography and passive torque during the first $(\checkmark)$ and the second $(\square)$ passive stretching tests for subjects 4 (A-) and 7 (B-) representing the two kinds of observed behavior.

Table 1

Coefficient of determination $\left(R^{2}\right)$ for the linear model fitted on LMH-torque relationships obtained during the first passive stretching test, and ratio between $\mathrm{LMH}_{\text {min }}$ and $\mathrm{LMH}_{\max }$

\begin{tabular}{lll}
\hline & $R^{2} P<0.001$ & $\mathrm{LMH}_{\max } / \mathrm{LMH}_{\min }$ \\
\hline Subject 1 & 0.834 & 1.84 \\
Subject 2 & 0.846 & 3.08 \\
Subject 3 & 0.707 & 2.86 \\
Subject 4 & 0.930 & 2.77 \\
Subject 5 & 0.825 & 2.95 \\
Subject 6 & 0.812 & 1.97 \\
Subject 7 & 0.856 & 2.72 \\
Subject 8 & 0.686 & 2.77 \\
Mean & 0.812 & 2.62 \\
SD & 0.080 & 0.46 \\
\hline
\end{tabular}

LMH: local muscle hardness.

SD: standard deviation.

\section{Discussion}

\subsection{LMH-angle and LMH-torque relationships}

In transient elastography, it is important to keep in mind that the displacement field is transient. Therefore, contrary to MRE techniques that uses stationary field, a 1-D measurement of a pulse wave along its direction of propagation gives an accurate estimation of the speed. However, in order to strictly investigate the nonisotropic structure of muscles, one option consists in controlling the wave polarization and the direction of propagation with respect to the main axis of anisotropy (Gennisson et al., 2003). Another option would be to measure the three components of a 3-D displacement field with an MRE device (e.g. Sinkus et al., 2005). As in previous papers (Dresner et al., 2001; Jenkyn et al., 2003; Heers et al., 2003), we have chosen a more global approach: the nonisotropic nature of the medium is ignored and the speed of a wave generated by a point source is supposed to reveal a global muscle hardness (LMH) that could be assessed by manual palpation. The present study was first designed to assess changes in this LMH with the stretching level. Results confirm the observation that could be performed by manual palpation, where the LMH is increased when the stretching level is increased. The $\mathrm{LMH}$-angle and $\mathrm{LMH}$-torque relationships obtained in passive conditions could be nicely modeled, respectively, using parabolic and linear fits. Determination coefficients obtained between passive torque and LMH were higher than those reported by Gennisson et al. (2005), between muscle activity and LMH during an incremental isometric contraction using the same shear elasticity probe $\left(R^{2}\right.$ of about 0.55 ). It is then probable that the contraction induces a greater variability than the passive stretching test because of spatial and temporal recruitment changes in muscles.

On average, the $\mathrm{LMH}_{\max } / \mathrm{LMH}_{\text {min }}$ ratio during the passive stretching test is $2.62 \pm 0.46$. As a comparison, Gennisson et al. (2005) obtained a similar $\mathrm{LMH}_{\max } /$ $\mathrm{LMH}_{\text {min }}$ ratio $(3.08 \pm 2.20)$ between the muscle at rest and muscle at $50 \%$ of the maximum voluntary contraction. The reason why the LMH increases with the level of stretching is not yet well known, but it could be assumed that collagen fibers are aligned in the direction of the stress when the muscle is stretched (Purslow, 1989) and that this phenomenon is partially responsible for the increase in the longitudinal stiffness of the muscle with the stretching (Gajdosik, 2001). This could be accompanied by an increase in the transverse stiffness, since a reduction in the muscle cross-section inducing a transverse stress and an increase in the intramuscular pressure (Davis et al., 2003) parallels the lengthening and the longitudinal stress induced by the stretching which could partly explain the increase in the LMH.

A linear relationship between the tension and LMH measured by MRE has been reported for an in vitro muscle (Dresner et al., 2001). Besides, using a specific hardness device with mechanical pressure, Murayama et al. (2005) confirmed that the transverse hardness is correlated with the passive tension of the isolated muscle ex vivo. Since the relationship between the level arm and the articular angle 
could be modeled by a linear function (Grieve et al., 1978; Hoang et al., 2005), a linear relationship between the passive torque at the ankle and the passive tension of the gastrocnemii could be considered in a first approximation. Therefore, our results which show a linear relationship between the passive torque and the LMH tend to confirm the linear relationship between muscle passive tension and passive $\mathrm{LMH}$.

\subsection{Reliability}

Tough test-retest studies require performing reproducible measurements, very little attention in the literature has been focused on the reliability of elastographic measurements performed in vivo. Previous studies on gel phantoms have shown that the transient elastography technique provides reproducible measurements (Gennisson et al., 2004; Gennisson and Cloutier, 2006). Results of the present study show that the reliability of the LMH changes during the stretching (slope of the LMH-torque relationship) is low. One main hypotheses could be proposed to explain this result. During the stretching, the muscle moves under the skin, whereas the shear elasticity probe was static. Various places of the muscle were then characterized during stretching, and heterogeneities in muscle could induce variability in LMH measurements. This hypothesis is strengthened by our result indicating that the LMH at rest (i.e. $y$-intercept of the LMH-torque relationship) is reliable. Therefore, the reliability of LMH measurements must be improved, and it would be interesting to use 2-D transient elastography (Bercoff et al., 2003; Sandrin et al., 2002a), 2-D ultrafast ultrasound imaging (Deffieux et al., 2006), or supersonic shear imaging (SSI) (Bercoff et al., 2004 ) in order to obtain an LMH mapping. SSI consists of using ultrasonic focused beams in order to generate mechanical vibration sources radiating low-frequency shear waves inside tissues without any external mechanical vibration (Bercoff et al., 2004). Therefore, it could be hypothesized that the use of this technique would decrease in the variability of the measurements, but its reliability will have to be determined.

Our results also showed that the reliability of the passive $\mathrm{LMH}$ at rest ( $y$-intercept of the LMH-torque relationship) is good. Therefore, the LMH at rest could be used to assess changes in muscle intrinsic stiffness following different solicitations. The better reliability of the $y$-intercept in comparison to the slope of $\mathrm{LMH}$-torque relationships could be easily explained by the lack of tissue motion for low torque levels inducing a lower variability in measurements.

\subsection{Effects of static stretching}

Considering reliability results, the effects of static stretching were assessed only using the $y$-intercept of the LMH-torque relationship (i.e. LMH at rest) and changes in the whole LMH-torque relationship have not been determined. Despite a significant decrease in passive torque after the static stretching protocol, our results showed that the passive LMH at rest was not significantly changed. Therefore, results of the present study tend to confirm results obtained from our previous hypothesis, that changes in passive torque following static stretching could be mainly explained by an acute increase in muscle length without any changes in intrinsic muscle mechanical properties. In isolated rabbit muscle, acute increases in muscle resting length have been demonstrated after 10 replicates of $30 \mathrm{~s}$ of static stretching (Taylor et al., 1990). These authors suggested that changes could be due to viscoelastic creep taking place within microstructures of both muscle and tendon tissues, but the relative contribution of these structures to the lengthening is not yet known.

\section{Conclusions}

Since transient elastography enables a quick assessment of localized muscle hardness in a noninvasive way and using simple protocols, LMH measurements would be of particular interest in the field for muscular biomechanics and myology. Our results show that the passive LMH measured in vivo increases during a passive stretching of the muscle, and LMH-torque relationship was nicely modeled by linear fits. Changes in the passive LMH during stretching are not reproducible, in contrast to the passive LMH at rest. No significant change in LMH at rest was found after the $10 \mathrm{~min}$ of static stretching protocol.

\section{Conflict of interest statement}

All authors disclose any financial and personal relationships with other people or organizations that could inappropriately influence this work.

\section{References}

Bercoff, J., Chaffai, S., Tanter, M., Sandrin, L., Catheline, S., Fink, M., Gennisson, J.L., Meunier, M., 2003. In vivo breast tumor detection using transient elastography. Ultrasound in Medicine and Biology 29, 1387-1396.

Bercoff, J., Tanter, M., Fink, M., 2004. Supersonic shear imaging: a new technique for soft tissue elasticity mapping. IEEE Transactions in Ultrasonics, Ferroelectronics, and Frequency Control 51, 396-409.

Catheline, S., Gennisson, J.L., Delon, G., Sinkus, R., Fink, M., Abouelkaram, S., Culioli, J., 2004. Measurement of viscoelastic properties of soft solid using transient elastography. Journal of the Acoustical Society of America 116, 3734-3741.

Cornu, C., Goubel, F., Fardeau, M., 1998. Stiffness of knee extensors in Duchenne muscular dystrophy. Muscle and Nerve 21, 1772-1774.

Cornu, C., Goubel, F., Fardeau, M., 2001. Muscle and joint elastic properties during elbow flexion in Duchenne muscular dystrophy. Journal of Physiology 533, 605-616.

Davis, J., Kaufman, K.R., Lieber, R.L., 2003. Correlation between active and passive isometric force and intramuscular pressure in the isolated rabbit tibialis anterior muscle. Journal of Biomechanics 36, 505-512.

Deffieux, T., Gennisson, J.L., Nordez, A., Tanter, M., Fink, M., 2006. Ultrafast imaging of in vivo muscle contraction using ultrasound. Applied Physics Letters 89, 184107-184113. 
Dresner, M.A., Rose, G.H., Rossman, P.J., Muthupillai, R., Manduca, A., Ehman, R.L., 2001. Magnetic resonance elastography of skeletal muscle. Journal of Magnetic Resonance Imaging 13, 269-276.

Gajdosik, R.L., 2001. Passive extensibility of skeletal muscle: review of the literature with clinical implications. Clinical Biomechanics 16, 87-101.

Gennisson, J.L., Cloutier, G., 2006. Sol-gel transition in Agar-gelatin mixtures studied with transient elastography. IEEE Transactions on Ultrasonics, Ferroelectrics and Frequency Control 53, 716-723.

Gennisson, J.L., Catheline, S., Chaffai, S., Fink, M., 2003. Transient elastography in anisotropic medium: application to the measurement of slow and fast shear wave speeds in muscles. Journal of the Acoustical Society of America 114, 536-541.

Gennisson, J.L., Baldeweck, T., Tanter, M., Catheline, S., Fink, M., Sandrin, L., Cornillon, C., Querleux, B., 2004. Assessment of elastic parameters of human skin using dynamic elastography. IEEE Transactions on Ultrasonics, Ferroelectrics and Frequency Control 51, 980-989.

Gennisson, J.L., Cornu, C., Catheline, S., Fink, M., Portero, P., 2005. Human muscle hardness assessment during incremental isometric contraction using transient elastography. Journal of Biomechanics 38, $1543-1550$.

Goubel, F., Lensel-Corbeil, G., 2003. Biomécanique. Eléments de mécanique musculaire, Paris, Masson.

Grieve, D., Phesant, S., Cavanagh, P.R., 1978. Prediction of gastrocnemius length from knee and ankle posture. In: Asmussen, E., Jorgensen, K. (Eds.), International Series of Biomechanics. pp. 405-412.

Hansen, P., Bojsen-Moller, J., Aagaard, P., Kjaer, M., Magnusson, S.P., 2006. Mechanical properties of the human patellar tendon, in vivo. Clinical Biomechanics 21, 54-58.

Heers, G., Jenkyn, T., Dresner, M.A., Klein, M.O., Basford, J.R., Kaufman, K.R., Ehman, R.L., An, K.N., 2003. Measurement of muscle activity with magnetic resonance elastography. Clinical Biomechanics 18, 537-542.

Hermens, H.J., Freriks, B., Disselhorst-Klug, C., Rau, G., 2000. Development of recommendations for SEMG sensors and sensor placement procedures. Journal of Electromyography and Kinesiology $10,361-374$.

Hewson, D.J., Hogrel, J.Y., Langeron, Y., Duchene, J., 2003. Evolution in impedance at the electrode-skin interface of two types of surface EMG electrodes during long-term recordings. Journal of Electromyography and Kinesiology 13, 273-279.

Hoang, P.D., Gorman, R.B., Todd, G., Gandevia, S.C., Herbert, R.D., 2005. A new method for measuring passive length-tension properties of human gastrocnemius muscle in vivo. Journal of Biomechanics 38, 1333-1341.

Hof, A.L., 1998. In vivo measurement of the series elasticity release curve of human triceps surae muscle. Journal of Biomechanics 31, 793-800.

Hopkins, W.G., 2000. Measures of reliability in sports medicine and science. Sports Medicine 30, 1-15.

Jenkyn, T.R., Ehman, R.L., An, K.N., 2003. Noninvasive muscle tension measurement using the novel technique of magnetic resonance elastography (MRE). Journal of Biomechanics 36, 1917-1921.

Kubo, K., Kanehisa, H., Kawakami, Y., Fukunaga, T., 2001. Influences of repetitive muscle contractions with different modes on tendon elasticity in vivo. Journal of Applied Physiology 91, 277-282.

Lambertz, D., Perot, C., Kaspranski, R., Goubel, F., 2001. Effects of long-term spaceflight on mechanical properties of muscles in humans. Journal of Applied Physiology 90, 179-188.
Levinson, S.F., Shinagawa, M., Sato, T., 1995. Sonoelastic determination of human skeletal muscle elasticity. Journal of Biomechanics 28, $1145-1154$.

Maganaris, C.N., Paul, J.P., 1999. In vivo human tendon mechanical properties. Journal of Physiology 521, 307-313.

Magnusson, S.P., Simonsen, E.B., Dyhre-Poulsen, P., Aagaard, P., Mohr, T., Kjaer, M., 1996. Viscoelastic stress relaxation during static stretch in human skeletal muscle in the absence of EMG activity. Scandinavian Journal of Medicine and Science in Sports 6, 323-328.

Magnusson, S.P., Aagard, P., Simonsen, E., Bojsen-Moller, F., 1998. A biomechanical evaluation of cyclic and static stretch in human skeletal muscle. International Journal of Sports Medicine 19, 310-316.

McNair, P.J., Dombroski, E.W., Hewson, D.J., Stanley, S.N., 2001. Stretching at the ankle joint: viscoelastic responses to holds and continuous passive motion. Medicine and Science in Sports Exercise 33, 354-358.

McNair, P.J., Hewson, D.J., Dombroski, E., Stanley, S.N., 2002. Stiffness and passive peak force changes at the ankle joint: the effect of different joint angular velocities. Clinical Biomechanics 17, 536-540.

Murayama, M., Yoneda, T., Kawai, S., 2005. Muscle tension dynamics of isolated frog muscle with application of perpendicular distortion. European Journal of Applied Physiology 93, 489-495.

Nordez, A., Cornu, C., McNair, P., 2006. Acute effects of static stretching on passive stiffness of the hamstring muscles calculated using different mathematical models. Clinical Biomechanics 21, 755-760.

Nordez, A., McNair, P.J., Casari, P., Cornu, C., in press. Acute changes in hamstrings musculo-articular dissipative properties induced by cyclic and static stretching. International Journal of Sports Medicine.

Ophir, J., Cespedes, I., Ponnekanti, H., Yazdi, Y., Li, X., 1991. Elastography: a quantitative method for imaging the elasticity of biological tissues. Ultrasonic Imaging 13, 111-134.

Pousson, M., Van Hoecke, J., Goubel, F., 1990. Changes in elastic characteristics of human muscle induced by eccentric exercise. Journal of Biomechanics 23, 343-348.

Purslow, P.P., 1989. Strain-induced reorientation of an intramuscular connective tissue network: implications for passive muscle elasticity. Journal of Biomechanics 22, 21-31.

Reeves, N.D., Maganaris, C.N., Narici, M.V., 2003. Effect of strength training on human patella tendon mechanical properties of older individuals. Journal of Physiology 548, 971-981.

Riemann, B.L., DeMont, R.G., Ryu, K., Lephart, S.M., 2001. The effects of sex, joint angle, and the gastrocnemius muscle on passive ankle joint complex stiffness. Journal of Athletic Training 36, 369-375.

Sandrin, L., Tanter, M., Catheline, S., Fink, M., 2002a. Shear modulus imaging with 2-D transient elastography. IEEE Transactions in Ultrasonics, Ferroelectronics, and Frequency Control 49, 426-435.

Sandrin, L., Tanter, M., Gennisson, J.L., Catheline, S., Fink, M., 2002 b. Shear elasticity probe for soft tissues with 1-D transient elastography. IEEE Transactions in Ultrasonics, Ferroelectronics, and Frequency Control 49, 436-446.

Sinkus, R., Tanter, M., Xydeas, T., Catheline, S., Bercoff, J., Fink, M., 2005. Viscoelastic shear properties of in vivo breast lesions measured by MR elastography. Magnetic Resonance Imaging 23, 159-165.

Taylor, C.D., Dalton, J.D., Seaber, A.V., Garrett, W.E., 1990. Viscoelastic properties of muscle-tendon units. The biomechanical effects of stretching. American Journal of Sports Medicine 18, 300-309.

Winters, J., L., S., Seif-Naraghi, A.H., 1988. An analysis of the sources of musculoskeletal system impedance. Journal of Biomechanics 21, 1011-1025. 